\title{
Construcción de modelos lógicos en las actividades bibliotecaria y de información
}

\author{
MigUel ÁNGELRENDÓN-ROJAS \\ SALVADORGORBEA-PORTAL \\ CentroUniversitariodeInvestigacionesBibliotecológicas, \\ UniversidadNacio nalAutóno madeMéxico,To rreII de \\ Humanidades, piso 12, CiudadUniversitaria, México,D.F. \\ Tel. (525) 623- 0341 Fax: (525) 550- 7461 \\ E-mail:marr@ servidor.unam.mx \\ CentroUniversitariodeInvestigacionesBibliotecológicas, \\ Universidad Nacio nalAutó no madeMéxico, To rreII de \\ Humanidades, piso 12,Ciu dad UniversitariaMéxico,D.F. \\ Tel. (525) 623- 0360 Fax: (525) 550- 7461 \\ E-mail:portal@ servidor.unam.mx
}

Trabajo

recibido el 15

deagosto de

1997

Trabajo

aceptado el 8

de enero de 1998

\section{RESUMEN}

Se destaca la importancia de la lógica como instrumento de modelación y se analizan los principios generales, condiciones iniciales y premisas que se observan en las actividades bibliotecaria y de información, con el propósito de construir modelos lógico-matemáticos que comprueben supuestos y ayuden a la explicación sobre el comportamiento de las tareas que la integran.

\section{ABSTRACT}

The importance of logic as an instrument in the development of models is stressed and the general principles, initial conditions and assumptions observed in librarianship and information activities, analysed. This is done with the purpose of constructing logic-mathematic models that confirm suppositions and help to explain the behaviour of the tasks that integrate them.

\section{INTRODUCCIÓN}

Duede tomarse por principio que una formulación matemática no constituye I por sí misma una teoría, sin embargo, cuando ésta representa una generalización de un fenómeno, o identifica el comportamiento de una regularidad, proceso u operación, o refleja determinadas relaciones no manifiestas a simple vista y, nunca antes comprobada, no cabe duda que se está en presencia de una aportación teórica que enriquece y genera nuevo conocimiento a la disciplina que lo recibe. 
También es evidente, por una parte, que el usuario de una biblioteca acude a ella con el propósito de satisfacer una necesidad de información y que el fondo de información y referencia y sus colecciones deben satisfacer esa necesidad, y por la otra, que esta necesidad puede variar como varían los fondos de información y referencia y sus colecciones, saber esto no es ciencia, porque no comprende las causas y condiciones que explican sus relaciones, sin embargo, cuando identificamos en estos fenómenos de fácil observación las causas y principios generales como premisas, que condicionan su existencia y se logra representarlas a través de formulaciones lógica o matemáticas, la comprensión y convencimiento sobre la presencia de un método científico no da lugar a dudas.

En el trabajo que se presenta a continuación se incursiona en la importancia de la lógica matemática como una efectiva herramienta en la modelación de principios y condiciones generales, cuya expresión manifiesta las premisas y relaciones de los componentes que determinan el funcionamiento de las actividades bibliotecarias y de información.

Poder formular lógica y matemáticamente los principios generales y relaciones que se dan en esta actividad o en el sistema de conocimientos que la representa, es sin lugar a dudas, una muestra fehaciente de que se está en presencia de una ciencia. Muchas han sido las demostraciones que a lo largo de la historia de la bibliotecología pudieran traerse a colación, como los modelos de Bradford, Burrell y las aplicaciones estadísticas de Ranganathan, sirva el presente trabajo como una tenue aportación que fortalezca en tal sentido el carácter científico de la bibliotecología y otras disciplinas del campo de la información.

\section{LA LÓGICA COMO INSTRUMENTO DE MODELACIÓN}

El término lógica posee diversos significados, en ocasiones es sinónimo de "coherencia", en otras de "evidente", y en unas más de "racional". Para evitar equívocos, aclararemos cuál es el sentido que le otorgamos a dicho término en el presente trabajo, y que podemos anticipar, se refiere a una ciencia formal que estudia las relaciones existentes entre determinadas estructuras abstractas.

En el campo de las ciencias encontramos dos teorías que llevan como nombre genérico el término lógica: la lóġa dialéticay la lóġa fomal. La primera estudia las leyes del desarrollo, cambio e interrelación de las formas del pensamiento; la segunda, que es la que por ahora nos interesa, estudia las leyes que rigen el razonamiento.

La lógica formal se suele dividir, sobre la base de criterios temporales, en tradicional y contemporánea. La primera abarca desde sus orígenes con Aristóteles hasta el siglo XIX, la segunda, también conocida como lógica matemática, fue el resultado de la aplicación de métodos propios de la matemática a la investigación lógica y sus orígenes se deben a los trabajos de Frege, Russell yWhitehead. En ellos se 
trata de formalizar el proceso de inferencia deductiva a través de la relación de las formas de los enunciados que son los elementos mínimos que pueden ser juzgados como verdaderos o falsos; de tal manera que si las premisas son verdaderas, es imposible deducir conclusiones falsas.

Estos sistemas descansan en dos principios fundamentales, el primero de los cuales consiste en el hecho de que todo enunciado o es verdadero o es falso, es decir, necesariamente tiene un valor de verdad, no existen enunciados sin valor de verdad; el segundo principio consiste en que todo enunciado tiene sólo un valor de verdad, no puede ser verdadero y falso a la vez. A este tipo de lógica basada en esos dos principios se le conoce como lógica dásica.

En el presente siglo se desarrolló rápidamente la lógica formal contemporánea y aparecieron diferentes tipos de lógicas como la lógica modal, la lógica polivalente, la lógica intuicionista, la lógica relevante, la lógica paraconsistente, la lógica no monotónica, la lógica difusa, entre otras. A estas disciplinas se les denomina lógicas nodásicas porque desechan al menos uno de los principios en los que se basa la lógica clásica o uno de los metateoremas que tiene lugar en la lógica clásica.

D e esta manera, la lógica a la que hacemos referencia en este trabajo se refiere a la lógica clásica formal contemporánea, la cual estudia las leyes que rigen la obtención de conocimientos verdaderos a partir de otros conocimientos verdaderos, esto es, las leyes que rigen la necesidad del paso de unos enunciados verdaderos a otros también verdaderos.

Es importante señalar que la lógica no estudia qué es la verdad y si esos enunciados de los que partimos son verdaderos y por qué lo son (de eso se encargará la teoría del conocimiento en el primer caso y las ciencias particulares en el segundo). Lo que hace la lógica como instrumento de trabajo intelectual es cuidar que no se pierda la verdad de los enunciados iniciales en el paso que se hace de cadenas de inferencias, esto es, podemos decir que "la lógica es el pastor de la verdad."

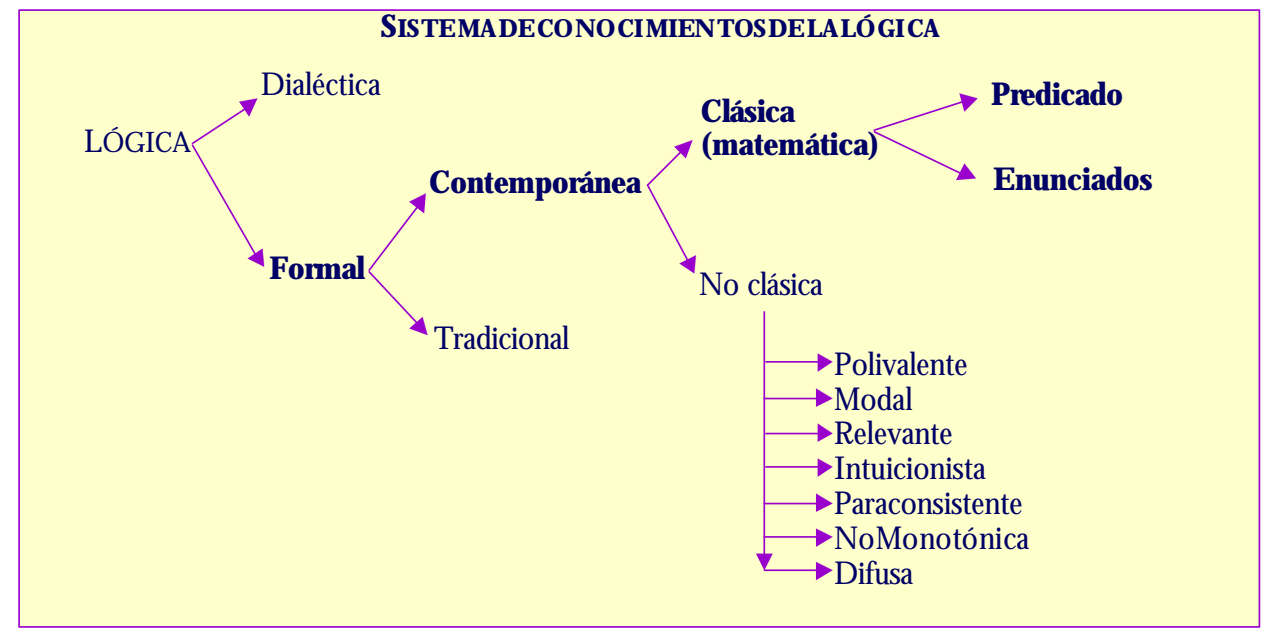


Como ciencia formal, la lógica utiliza un lenguaje que tiene como finalidad reflejar y hacer evidente las estructuras (formas) de los objetos analizados (enunciados), las cuales al combinarse y transformarse permiten en ese nivel estructural (formal), abstrayéndose de todo contenido, garantizar las inferencias correctas y obstruir toda posibilidad de unas inferencias incorrectas.

El lenguaje utilizado por la lógica se caracteriza por tener una sintaxis precisa que, en primer lugar, expresa de una manera clara, explícita y completa los símbolos iniciales que se van a emplear (alfabeto), en segundo lugar, se dan también de una manera explícita y estricta (algorítmica) las reglas de formación de palabras a partir de los símbolos iniciales y en tercer lugar, se dan del mismo modo que las anteriores, las reglas de transformación de palabras (reglas de inferencia).

Existen dos clases de sistemas lógicos según las maneras en que se transforman las expresiones: axiomáticas o de cálculo natural, la primera consiste en que a partir de un conjunto finito de enunciados primitivos se derivan todas las demás expresiones (teoremas) con ayuda de pocas reglas de inferencia; el sistema de cálculo natural consiste en que en lugar de axiomas se toma un mayor número de reglas de inferencia.

En la lógica clásica contemporánea existen dos sistemas según el nivel de análisis que se realice de los enunciados, si sólo se contempla el enunciado en sí como una totalidad, sin adentrarse en la estructura interna, se tiene la lógica de enunciados; si por el contrario, se analiza la estructura del mismo enunciado, se tiene la lógica de predicados. La última es la expansión de la primera porque la incluye y además posee sus símbolos, reglas y teoremas específicos. D ebido a que en el presente trabajo emplearemos el lenguaje de la lógica formal tanto de enunciados como de predicados daremos a continuación una breve descripción de ellos, según Mendelson (1987).

\section{Lenguaje de la lógica de enunciados (L. E.)}

Sintaxis del lenguaje de la lógica de enunciados para el sistema axiomático

\section{Alfabeto}

A) Símbolos para representar enunciados (letras enunciativas).

$\mathrm{P}, \mathrm{Q}, \mathrm{R}, \mathrm{S}, \mathrm{T}, \mathrm{U}, \mathrm{V}, \mathrm{P}_{1}, \mathrm{Q}_{1}, \mathrm{R}_{1}, \mathrm{~S}_{1}, \mathrm{~T}_{1}, \mathrm{U}_{1}, \mathrm{~V}_{1}, \mathrm{P}_{2}, \mathrm{Q}_{2}, \mathrm{R}_{2}, \mathrm{~S}_{2}, \mathrm{~T}_{2}, \mathrm{U}_{2}, \mathrm{~V}_{2}, \mathrm{P}_{3}, \mathrm{Q}_{3}$, $R_{3}, S_{3}, T_{3}, U_{3}, V_{3}, \ldots P_{n}, Q_{n}, R_{n}, S_{n}, T_{n}, U_{n}, V_{n}, \ldots$

B) Símbolos para constantes lógicas

$\neg$ (Negación, "no"), \& (Conjunción, “y"), $\vee($ Disyunción, "0"),

$\supset$ (Implicación, "si ... entonces"), $\equiv($ Bicondicional, "si y sólo si").

C) Símbolos técnicos

Paréntesis izquierdo "("; paréntesis derecho ")". 


\section{Fómula bien formada}

1. Toda letra enunciativa es fórmula bien formada.

2. Si A y B son fórmulas bien formadas, entonces $(\neg A),(A \& B),(A \vee B),(A \supset B)$, $(\mathrm{A} \equiv \mathrm{B})$ son fórmulas bien formadas.

3. Ninguna otra expresión, a excepción de los puntos 1 y 2 de esta definición, es fórmula bien formada.

\section{Definición}

$$
(\mathrm{A} \equiv \mathrm{B})={ }_{\mathrm{DEF}} .(\mathrm{A} \supset \mathrm{B}) \&(\mathrm{~B} \supset \mathrm{A})
$$

Si es un sistema axiomático:

\section{Axiomas}

1. $(\mathrm{A} \supset(\mathrm{B} \supset \mathrm{C})) \supset((\mathrm{A} \supset \mathrm{B}) \supset(\mathrm{A} \supset \mathrm{C}))$

2. $(\mathrm{A} \supset(\mathrm{B} \supset \mathrm{A}))$

3. $A \supset(B \supset(A \& B))$

4. $(\mathrm{A} \& \mathrm{~B}) \supset \mathrm{A}$

5. $(\mathrm{A} \& \mathrm{~B}) \supset \mathrm{B}$

6. $A \supset(A \vee B)$

7. $\mathrm{B} \supset(\mathrm{A} \vee \mathrm{B})$

8. $(A \vee B) \supset(\neg B \supset A)$

9. $(\mathrm{A} \supset \mathrm{B}) \supset((\mathrm{A} \supset \neg \mathrm{B}) \supset \neg \mathrm{A})$

10. $\neg \neg \mathrm{A} \supset \mathrm{A}$

\section{Reglas de inferencia}

$(A \supset B)$

$$
\frac{\mathrm{A}}{\mathrm{B}} \quad \text { Modusponens }
$$

Si se construye el lenguaje de la lógica de enunciados para un sistema de cálculo natural, la sintaxis es semejante a la del sistema axiomático en los puntos I, II y III (en el alfabeto, la definición de fórmula bien formada y definición de la equivalencia), diferenciándose de que en lugar de IV y V (axiomas y modus ponens) se tienen las siguientes reglas de inferencia:

\section{Reglas de inferencia para el sistema de cálculo natural}

$$
\text { 1. } \frac{\mathrm{A}}{\neg \neg \mathrm{A}} \quad \text { 2. } \frac{\neg \neg \mathrm{A}}{\mathrm{A}} \quad \text { 3. } \frac{\mathrm{A}}{\frac{\mathrm{B}}{\mathrm{A} \& \mathrm{~B}}} \quad \text { 4. } \frac{\mathrm{A} \& \mathrm{~B}}{\mathrm{~A}}
$$



5. $\frac{\mathrm{A} \& \mathrm{~B}}{\mathrm{~B}}$
6. $\frac{A}{A \vee B}$
7. $\frac{B}{A \vee B}$
8. $\frac{A \vee B}{\frac{\neg A}{B}}$
9. $\frac{\mathrm{A} \vee \mathrm{B}}{\frac{\neg \mathrm{B}}{\mathrm{A}}}$
10. $\frac{\mathrm{A} \supset \mathrm{B}}{\frac{\mathrm{A}}{\mathrm{B}}}$
11. $\frac{\mathrm{A} \supset \mathrm{B}}{\frac{\neg \mathrm{B}}{\neg \mathrm{A}}}$
12. $\frac{B}{A \supset B}$

\section{Lenguaje de la lógica de predicados de primer orden}

\section{Sintaxis del lenguaje de la lógica de predicados de primer orden para el sistema axiomático}

\section{Alfabeto}

A) Símbolos del L.E.

B) Símbolos para constantes individuales (sustantivos propios).

$a, b, c, d, a_{1}, b_{1}, c_{1}, d_{1}, a_{2}, b_{2}, c_{2}, d_{2}, a_{3}, b_{3}, c_{3}, d_{3}, \ldots, a_{n}, b_{n}, c_{n}, d_{n}, \ldots$

C) Símbolos para variables individuales (sustantivos comunes).

$\mathrm{x}, \mathrm{y}, \mathrm{z}, \mathrm{x}_{1}, \mathrm{y}_{1}, \mathrm{z}_{1}, \mathrm{x}_{2}, \mathrm{y}_{2}, \mathrm{z}_{2}, \mathrm{x}_{3}, \mathrm{y}_{3}, \mathrm{z}_{3}, \ldots, \mathrm{x}_{\mathrm{n}}, \mathrm{yn}_{\mathrm{n}}, \mathrm{z}_{\mathrm{n}}, \ldots$

D) Símbolos para predicados (propiedades y relaciones de y entre constantes y variables individuales)

$\mathrm{P1}_{1}, \mathrm{Q}_{1}, \mathrm{R}_{1}{ }_{1}, \mathrm{~S}_{1}, \mathrm{~T}_{1}, \mathrm{U}_{1}, \mathrm{~V}_{1}, \mathrm{P}_{2}, \mathrm{Q}_{2}, \mathrm{R}_{2}, \mathrm{~S}_{2}, \mathrm{~T}_{2}, \mathrm{U}_{2}, \mathrm{~V}_{2}, \mathrm{P}_{3}, \mathrm{Q}_{3}, \mathrm{R}_{3}, \mathrm{~S}_{3}$, $\mathrm{T}_{3}, \mathrm{U}_{3}, \mathrm{~V}_{3}, \ldots, \mathrm{P}_{\mathrm{n}}, \mathrm{Q}{ }_{\mathrm{n}}, \mathrm{R}_{\mathrm{n}}, \mathrm{S}_{\mathrm{n}}, \mathrm{T} 1_{\mathrm{n}}, \mathrm{U} 1_{\mathrm{n}}, \mathrm{V}^{1}{ }_{\mathrm{n}}, \ldots$

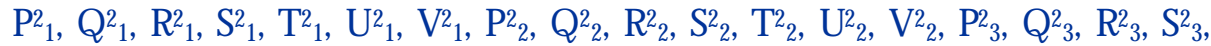
$\mathrm{T}_{2}, \mathrm{U}_{2}, \mathrm{~V}_{2}, \ldots, \mathrm{P}_{\mathrm{n}}, \mathrm{Q}^{2}, \mathrm{R}_{\mathrm{n}}, \mathrm{S}_{\mathrm{n}}, \mathrm{T}_{\mathrm{n}}, \mathrm{U}^{2}, \mathrm{~V}^{2}, \ldots$

$\mathrm{P}_{1}, \mathrm{Q}^{3}, \mathrm{R}_{3}, \mathrm{~S}_{1}, \mathrm{~T}_{1}, \mathrm{U}_{1}, \mathrm{~V}_{1}, \mathrm{P}_{2}, \mathrm{Q}_{2}, \mathrm{R}_{2}, \mathrm{~S}_{2}, \mathrm{~T}_{2}, \mathrm{U}_{2}, \mathrm{~V}_{2}, \mathrm{P}_{3}, \mathrm{Q}_{3}, \mathrm{R}_{3}, \mathrm{~S}_{3}$, $\mathrm{T}_{3}, \mathrm{U}_{3}, \mathrm{~V}_{3}, \ldots, \mathrm{P}_{\mathrm{n}}, \mathrm{Q}^{3} \mathrm{n}, \mathrm{R}_{\mathrm{n}}, \mathrm{S}_{\mathrm{n}}, \mathrm{T}_{\mathrm{n}}, \mathrm{U}^{3}, \mathrm{~V}^{3}{ }_{\mathrm{n}}, \ldots$

$\mathrm{Pn}_{1}, \mathrm{Qn}_{1}, \mathrm{Rn}_{1}, \mathrm{Sn}_{1}, \mathrm{Tn}_{1}, \mathrm{Un}_{1}, \mathrm{Vn}_{1}, \mathrm{Pn}_{2}, \mathrm{Qn}_{2}, \mathrm{Rn}_{2}, \mathrm{Sn}_{2}, \mathrm{Tn}_{2}, \mathrm{Un}_{2}, \mathrm{Vn}_{2}, \mathrm{Pn}_{3}, \mathrm{Qn}_{3}, \mathrm{Rn}_{3}, \mathrm{~S}$ $\mathrm{n}_{3}, \mathrm{~T} \mathrm{n}_{3}, \mathrm{Un}_{3}, \mathrm{Vn}_{3}, \ldots, \mathrm{Pn}_{\mathrm{n}}, \mathrm{Qn}_{\mathrm{n}}, \mathrm{R}_{\mathrm{n}}, \mathrm{Sn}_{\mathrm{n}}, \mathrm{Tn}_{\mathrm{n}}, \mathrm{Un}_{\mathrm{n}}, \mathrm{Vn}_{\mathrm{n}}, \ldots$

E) Símbolos para constantes lógicas

Los símbolos del L.E: y $\forall$ (Cuantificador Universal, "todos"), $\exists$ (Cuantificador Existencial, "algún o algunos").

F) Símbolos técnicos

Paréntesis izquierdo y derecho; y la coma "," 


\section{Fómula bien formada}

A) D efinición de término:

1. Toda constante individual $a_{i}$ es término.

2. Toda variable individual $\mathrm{x}_{\mathrm{i}}$ es término.

3. Ninguna otra expresión, a excepción de los puntos 1 y 2 de esta definición, es término.

B) D efinición de fórmula bien formada

0. Las reglas del L.E.

1. Si $P_{k}$ es un predicado y $t_{1}, t_{2}, t_{3}, \ldots, t_{j}$ son términos, entonces $P_{j_{k}}\left(t_{1}, t_{2}, t_{3}, \ldots, t_{j}\right)$ es fórmula bien formada.

2. Si A es fórmula bien formada y $\mathrm{x}_{\mathrm{i}}$ es variable individual, entonces $\forall \mathrm{x}_{\mathrm{i}} \mathrm{A}$ y $\exists \mathrm{x}_{\mathrm{i}}$ A son fórmulas bien formadas.

3. Ninguna otra expresión, a excepción de los puntos 1, 2 y 3 de esta definición, es fórmula bien formada.

Si es sistema axiomático:

\section{Definición}

$$
\exists \mathrm{xA}(\mathrm{x})=\text { Def. } \neg \forall \mathrm{x} \neg \mathrm{A}(\mathrm{x})
$$

\section{Axiomas}

0 . Axiomas de la lógica de enunciados

1. $\forall \alpha \mathrm{A}(\alpha) \supset \mathrm{A}\left(\mathrm{t}_{\mathrm{n}}\right)$

\section{Reglas de inferencia}

Las propias del L.E y $\frac{\mathrm{A}(\alpha)}{\forall \alpha \mathrm{A}(\alpha)}$ donde $\alpha$ está limitada

La sintaxis del lenguaje de la lógica de predicados de primer orden para cálculo natural es análoga en su alfabeto, noción de fórmula y definición del cuantificador existencial (puntos I, II y III) a la sintaxis del lenguaje del sistema axiomático, con la diferencia de que en lugar de axiomas y reglas de inferencia (puntos IV y V) se trabajó únicamente con las siguientes reglas de inferencia:

\section{Reglas de inferencia para el sistema de cálculo natural de la lógica de} predicados

0. Las reglas de la lógica de enunciados y

1. $\frac{\forall \alpha \mathrm{A}(\alpha)}{\mathrm{A}(\alpha)} \quad$ 2. $\frac{\mathrm{A}(\alpha)}{\forall \alpha \mathrm{A}(\alpha)}$ donde $\alpha$ está limitada 
3. $\frac{\exists \alpha \mathrm{A}(\alpha)}{\mathrm{A}(\alpha)}$ donde $\alpha$ está limitada $\quad$ 4. $\frac{\mathrm{A}(\alpha)}{\exists \alpha \mathrm{A}(\alpha)}$

D espués de haber dado el lenguaje de los sistemas lógicos veamos cómo este instrumento sirve de modelación en la actividad científica, principalmente para explicar y predecir [pronosticar]. Según el modelo clásico de Hempel, explicar es deducir lógicamente a partir de leyes generales y condiciones iniciales lo que se explica, si se tiene ya presente lo explicado; y predecir [pronosticar]es esa misma operación, pero cuando aún no está presente lo que se explica (Hempel, 1979).

De esta manera tenemos:

LEYES GENERALES

CONDICIONESINICIALES

(D onde la raya horizontal indica

EXPLICACIÓN (PRONÓSTICO) una deducción lógica)

Podemos ejemplificar el esquema anterior con la siguiente situación:

Tenemos tres leyes de las ciencias naturales:

* El agua se congela cuando la temperatura baja de $0^{\circ} \mathrm{C}$.

* El volumen del agua aumenta en su estado sólido.

* Todo metal posee un coeficiente de resistencia y si se rebasa ese nivel, se rompe.

y cuatro condiciones iniciales:

* El radiador del auto contiene agua.

* El radiador es de metal.

- La temperatura durante la noche descendió a bajo 0 o C.

* La presión ejercida por el agua congelada en el radiador es mayor al Coeficiente de resistencia del material que lo contiene.

A partir de esas siete premisas ¿qué podemos concluir?

Formalicemos el anterior argumento primero simbolizando sus componentes de la siguiente manera:

$\mathrm{P}(\mathrm{)})=$ Ser agua .

$\mathrm{Q}\left(\mathrm{)}=\right.$ Tener temperatura bajo $0^{0} \mathrm{C}$.

$\mathrm{R}(\mathrm{)})$ Congelarse.

$\mathrm{S}(\mathrm{)}=$ Ser metal.

$\mathrm{P}_{1}(\mathrm{)})=$ Aumentar volumen.

$\mathrm{Q}_{1}(\mathrm{)})=$ Tener un coeficiente de resistencia.

$\mathrm{R}_{1}(\mathrm{)})=$ Superar el coeficiente de resistencia.

$\mathrm{S}_{1}(\mathrm{)})=$ Romperse . 
y posteriormente realicemos la siguiente traducción de las premisas iniciales:

LEYES

$$
\mid \begin{aligned}
& \forall \mathrm{x}((\mathrm{P}(\mathrm{x}) \& \mathrm{Q}(\mathrm{x})) \supset \mathrm{R}(\mathrm{x})) \\
& \forall \mathrm{x}\left((\mathrm{P}(\mathrm{x}) \& \mathrm{R}(\mathrm{x})) \supset \mathrm{P}_{1}(\mathrm{x})\right) \\
& \forall \mathrm{x}\left(\left(\mathrm{S}(\mathrm{x}) \supset \mathrm{Q}_{1}(\mathrm{x})\right) \&\left(\mathrm{R}_{1}(\mathrm{x}) \supset \mathrm{S}_{1}(\mathrm{x})\right)\right)
\end{aligned}
$$

CONDICIONES

INICIALES

$$
\begin{aligned}
& \mathrm{Q}(\mathrm{a}) \\
& \left(\mathrm{R}(\mathrm{a}) \supset \mathrm{P}_{1}(\mathrm{a})\right) \&\left(\mathrm{P}_{1}(\mathrm{a}) \supset \mathrm{R}_{1}(\mathrm{a})\right)
\end{aligned}
$$

y a partir de las cuales obtendremos:

$\mathrm{S}_{1}(\mathrm{a})$

Esto es, que el radiador se rompió (o romperá sí aún no sabemos ese hecho).

Si en lugar de leyes de las ciencias naturales colocamos leyes bibliotecológicas, y en condiciones iniciales ponemos situaciones concretas de la actividad bibliotecaria y de información, como por ejemplo la aplicación que hace Rijsbergen (1996) de la lógica a la recuperación de información, entonces este mismo esquema puede ser empleado para el análisis de dicha actividad y consecuentemente nos puede servir para modelarla. Esto es un gran reto porque por un lado, nos impulsa a investigar y descubrir leyes o regularidades en este campo, y por otro lado, nos conduce a asentar situaciones específicas como condiciones iniciales, lo que significa en el primer caso realizar una investigación teórica y en el segundo, un estudio empírico.

\section{MODELACIÓN LÓGICO-MATEMÁTICA DE LAS ACTIVIDADES BIBLIOTECARIA Y DE INFORMACIÓN}

Las actividades bibliotecarias y de información surgen a partir de la necesidad del hombre por informarse, a lo largo de la historia de la sociedad se han desarrollado al grado tal que, tanto para el trabajador intelectual contemporáneo, permanente usuario de los servicios de información, como para el profesional moderno, éstas constituyen actividades científicas debido a que los métodos de trabajo que emplean son científicos. Sin embargo, es común encontrar profesionales que opinan lo contrario, dado que en sus inicios sus métodos eran fundamentalmente empíricos.

En la actualidad, estas actividades se apoyan en un sistema de conocimientos, conformado por las disciplinas biológico - informativas (bibliotecología, bibliografología, archivología y la ciencia de la información), que de manera interdisciplinaria se relacionan entre sí. Este sistema de conocimiento, a su vez, se ha relacionado de igual forma con otras disciplinas científicas pertenecientes a su propia 
esfera, es decir, la social, como la economía, la sociología, la psicología, la filosofía y otras de diferentes esferas como la matemática, la cibernética, la computación, entre otras.

Las relaciones interdisciplinarias han contribuido a que estas actividades apliquen en el desempeño de sus funciones, cada vez con mayor fuerza, novedosas teorías como la de sistema, la matemática de la información, las de las telemáticas, la de la comunicación científica, y otras provenientes de la economía, la sociología, la psicología por mencionar sólo algunos ejemplos.

Lo anterior ha provocado que las actividades bibliotecaria y de información constituyan en la actualidad actividades complejas en las que se requiere implantar en sus funcionamientos, gestiones y estudios, métodos científicos debido al nivel de complejidad que han alcanzado los fenómenos que se generan de sus procesos, tareas y operaciones unitarias. Ello ha propiciado también que sean analizadas como un sistema, modeladas y simuladas matemáticamente, en lo general y particular de sus funciones.

Si se consideran tales premisas, resulta factible también pensar que la lógica formal contemporánea y más específicamente la clásica o matemática pueda constituir una valiosa herramienta que facilite su explicación y pronóstico.

Sería conveniente entonces, empezar por definir en sentido muy general las actividades bibliotecaria y de información, así como las etapas o componentes principales que la integran. Éstas son consideradas como una esfera de la actividad económica dirigida a satisfacer las necesidades de información científica y técnica de los usuarios, integrada por las tareas siguientes:

* Recolección: Obtención de la información y completamiento de los Fondos de Información y Referencia, así como del desarrollo de sus colecciones.

* Procesamiento Analítico - Sintético: Proceso de análisis y síntesis de la información contenida en los documentos científicos y técnicos, mediante el cual se confeccionan e indizan anotaciones, resúmenes, reseñas, descripciones bibliográficas.

* Almacenamiento (Conservación): Organización y conservación de colecciones y Fondos de Información y Referencia.

* Búsqueda: Organización de la búsqueda informativa por medios manuales 0 automáticos, mediante accesos remotos o locales.

* Difusión: Hacer llegar la información al usuario con el apoyo de las otras tareas.

A la luz de un enfoque sistémico, esta última tarea, la difusión, es la que cumple la función ostensible del sistema, por lo que se considera la más importante de estas actividades, en tal sentido las restantes tareas se consideran de apoyo.

La formulación lógica de este supuesto general bien puede enunciarse tal como sigue: 
$(\neg \mathrm{R} \supset \neg \mathrm{P}) \&(\neg \mathrm{P} \supset \neg \mathrm{Q}) \&(\neg \mathrm{Q} \supset \neg \mathrm{S}) \&(\neg \mathrm{S} \supset \neg \mathrm{T})$

donde: $\quad \mathrm{R}=$ Hay recolección

$\mathrm{P}=$ Hay procesamiento

$\mathrm{A}=$ Hay almacenamiento

$\mathrm{B}=0$ curre búsqueda

$\mathrm{D}=$ Se da la difusión

La recolección, el procesamiento, el almacenamiento, y la búsqueda son condiciones necesarias para la difusión.

O tro componente importante del sistema lo constituye el usuario de la información, definido como la persona o colectivo que utiliza o puede utilizar los servicios de información científica y técnica. A los usuarios se les puede considerar como: individuales, colectivos, reales o potenciales; y constituyen el ente activo del ciclo social de la información (generación-uso), al mismo tiempo son generadores y "consumidores" de información.

\section{E tapas de las actividades bibliotecaria y de información y su relación con el ciclo social de la información}

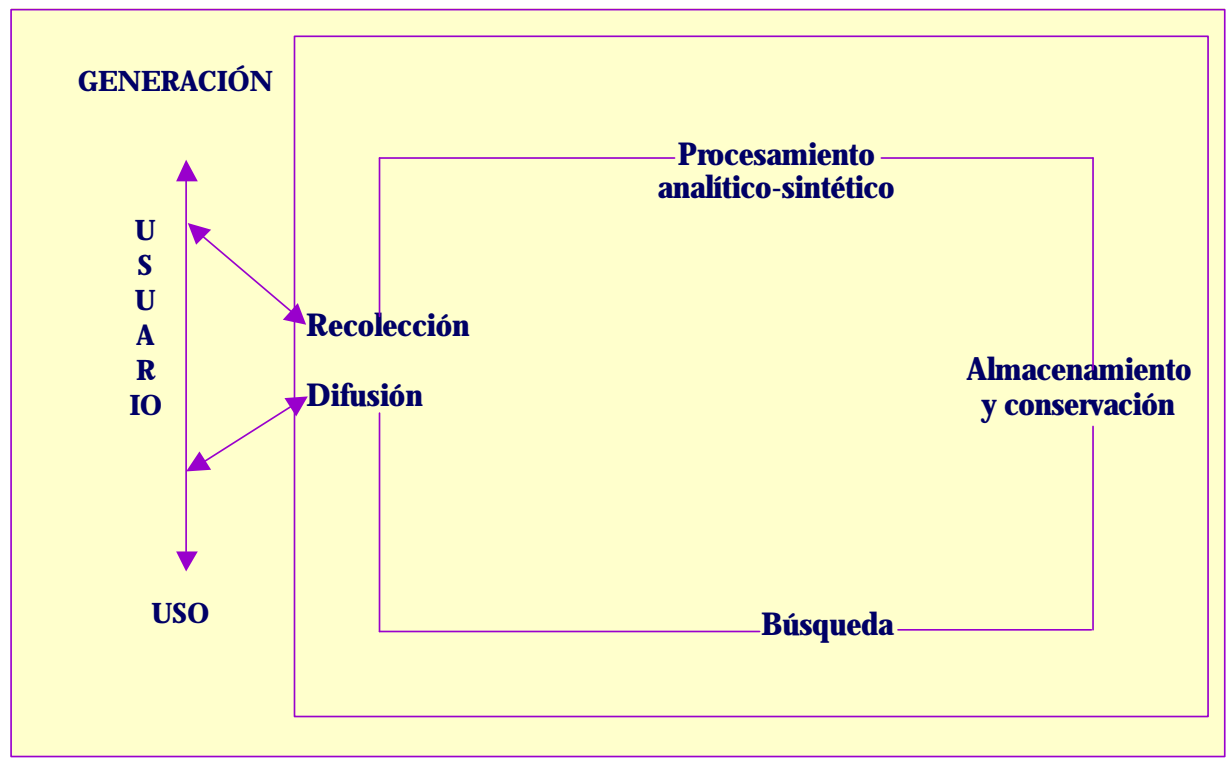

Como se puede observar, el nivel de complejidad de estas actividades no sólo se debe a sus componentes, estructuras y relaciones, sino también a su naturaleza y vinculación con fenómenos sociales, que en cierta medida lo rigen y condicionan, como sucede con el ciclo social de la información. 
Este vínculo y compromiso social requiere que el desarrollo de estas actividades se fundamente en determinados principios y condiciones generales como premisas que garanticen su gestión en términos de calidad y eficiencia. Estos principios en sentido general pueden estar asociados con:

* Inversión mínima de los recursos humanos y materiales en la organización de las actividades bibliotecaria y de información.

* Presentación de la información científico-técnica en plazos óptimos (exacta, completa y oportuna).

Los anteriores principios se pueden formular en lenguaje lógico de la forma siguiente:

Sí: $\quad \mathrm{P}(\mathrm{)}=$ Ser organización de las actividades bibliotecaria y de información.

$Q($ ) = Ser recursos humanos.

$\mathrm{R}($ ) $=$ Ser recursos materiales.

I ( ) = Existir cantidad mínima de recursos.

Entonces:

$\forall \mathrm{x} \forall \mathrm{y} \forall \mathrm{z}((\mathrm{P}(\mathrm{x}) \& \mathrm{Q}(\mathrm{y}) \& \mathrm{R}(\mathrm{z})) \supset((\mathrm{P}(\mathrm{x}) \supset(\mathrm{I}(\mathrm{y}) \& \mathrm{I}(\mathrm{z})))))$

Sí: $\quad \mathrm{P}(\mathrm{)}=\mathrm{Ser}$ información científico - técnica presentada.

Q ( ) =Ser información presentada en forma exacta.

$\mathrm{R}$ ( ) =Ser información presentada en forma completa.

S ( ) =Ser información presentada en forma oportuna.

Entonces:

$\forall \mathrm{x}((\mathrm{P}(\mathrm{x}) \supset(\mathrm{Q}(\mathrm{x}) \& \mathrm{R}(\mathrm{x}) \& \mathrm{~S}(\mathrm{x})))$

Para el cumplimiento de tales principios será necesario contar con las condiciones generales siguientes:

* Coordinación e interacción entre las diferentes unidades de información de perfil afín y otras del sistema nacional.

* Correspondencia entre la información emitida respecto a las demandas y necesidades de información de los usuarios del sistema.

Sí: $\quad \mathrm{P}(\mathrm{)}=\mathrm{Ser}$ unidad de información de perfil afín.

$\mathrm{Q}($ ) = Ser unidad de información del sistema nacional.

$\mathrm{R}()=$, Tener correspondencia entre.

$\mathrm{S}()=$, Tener interacción entre.

Entonces:

$\forall \mathrm{x} \forall \mathrm{y}((\mathrm{P}(\mathrm{x}) \& \mathrm{Q}(\mathrm{y})) \supset(\mathrm{R}(\mathrm{x}, \mathrm{y}) \& \mathrm{~S}(\mathrm{x}, \mathrm{y})))$ 
Sí: $\quad \mathrm{P}(\mathrm{)}=\mathrm{Ser}$ información emitida.

$Q($ ) = Ser demanda de información.

$\mathrm{C}()=$, Haber correspondencia entre.

Entonces:

$$
\forall \mathrm{x} \forall \mathrm{y}((\mathrm{P}(\mathrm{x}) \& \mathrm{Q}(\mathrm{y})) \supset \mathrm{C}(\mathrm{x}, \mathrm{y})))
$$

Pero no sólo estos principios generales y condiciones iniciales garantizan una óptima organización de las actividades y servicios bibliotecarios y de información, sino que su perfeccionamiento requiere considerar otras condiciones para su funcionamiento, tales como:

* Estudio constante de las demandas informativas.

* Organización de los flujos de información documental.

* Organización y utilización de los fondos de información y referencia y desarrollo de sus colecciones.

* Elaboración y utilización del sistema de búsqueda y referencia.

* Presencia de medios técnicos.

* Personal calificado.

Un análisis sistémico en las tareas y funciones de estas actividades facilita la modelación lógica de aquellas condiciones iniciales y premisas más específicas, lo que contribuye a su explicación y facilita el pronóstico de su comportamiento.

Tomemos como muestra algunas condiciones iniciales presentes en ciertas tareas:

\section{Condiciones iniciales}

Recolección:

Conservación:

El documento "a" cuesta X \$.

El documento " $\mathrm{b}$ " debe estar en las condiciones $\mathrm{X}$ para que no se destruya.

Procesamiento:

El documento " $\mathrm{C}$ " debe estar con la etiqueta $\mathrm{X}$, en el lugar $\mathrm{Y}$, etcétera.

Difusión:

Para satisfacer los servicios $\mathrm{x}, \mathrm{y}, \mathrm{z}$, se debe contar con los documentos a, b, c, el suficiente personal calificado $R$, y el mobiliario adecuado $\mathrm{S}$.

Estas condiciones iniciales pueden estar asociadas con las premisas siguientes:

\section{Premisas}

1. Si aumentan los recursos, aumenta la colección.

2. Si aumentan los recursos, aumenta el personal. 
3. Si aumentan los recursos, se mejora el mobiliario.

4. Si se aumenta la colección, el personal y se mejora el mobiliario, puede aumentar la eficiencia de la biblioteca.

Si aumentan los recursos puede aumentar la eficiencia de la biblioteca

(Condición inicial: el director de la biblioteca emplea los recursos para lo indicado en 1, 2 y 3).

\section{Simbolización}

P: Los recursos aumentan.

Q: La colección aumenta.

R: El personal aumenta.

S: El mobiliario se mejora.

$\mathrm{T}$ : La eficacia puede aumentar.

\section{Modelación lógica}

1. $\mathrm{P} \supset \mathrm{Q}$

2. $\mathrm{P} \supset \mathrm{R}$

3. $\mathrm{P} \supset \mathrm{S}$

4. $(\mathrm{Q} \& R \& S) \supset \mathrm{T}$

$\mathrm{P} \supset \mathrm{T}$

\section{Demostración}

\begin{tabular}{|c|c|}
\hline 1. $P \supset Q$ & Premisa \\
\hline 2. $\mathbf{P} \supset \mathbf{R}$ & Premisa \\
\hline 3. $\mathrm{P} \supset \mathrm{S}$ & Premisa \\
\hline 4. $(Q \& R \& S) \supset T$ & Premisa \\
\hline 5. $\mathrm{P}$ & Premisa adicional \\
\hline 6. Q & de 1 y 5 por $\supset$ e \\
\hline 7. $\mathrm{R}$ & de 2 y 5 por $\supset$ e \\
\hline 8.S & de 3 y 5 por $\supset e$ \\
\hline 9. $(Q \& R \& S)$ & de $6,7 y 8$ por $\& y$ \\
\hline 10. $\mathrm{T}$ & de 4 y 9 por $\supset$ e \\
\hline 11. $\mathrm{P} \supset \mathrm{T}$ & de $5-10$ por $\supset \mathrm{i}$ \\
\hline
\end{tabular}

1. Si aumenta la adquisición, aumentan los préstamos.

2. Si aumentan los préstamos, aumenta la circulación de documentos.

Si aumenta la adquisición, aumenta la circulación de documentos. 


\section{Simbolización}

P: La adquisición aumenta.

Q: Los préstamos aumentan.

$\mathrm{R}$ : La circulación aumenta.

Formalización del argumento

$$
\begin{aligned}
& \text { 1. } \mathrm{P} \supset \mathrm{Q} \\
& \text { 2. } \mathrm{Q} \supset \mathrm{R}
\end{aligned}
$$

$$
\mathrm{P} \supset \mathrm{R}
$$

\section{Demostración}

\begin{tabular}{|l|l|}
\hline 1. $P \supset \mathbf{Q}$ & Premisa \\
\hline 2. $\mathbf{R} \mathbf{R}$ & Premisa \\
\hline 3. $P$ & $\begin{array}{l}\text { Premisa } \\
\text { adicional }\end{array}$ \\
\hline 4. $Q$ & de 1y 3 por $\supset \mathbf{e}$ \\
\hline 5. $\mathbf{R}$ & de 2y 4 por $\supset \mathbf{e}$ \\
\hline 6. $P \supset \mathbf{R}$ & de 3-5 por $\supset \mathbf{i}$ \\
\hline
\end{tabular}

Lo anterior demuestra que el análisis sistémico permite establecer premisas y condiciones iniciales, que pueden ser modeladas lógicamente, para cada tarea, proceso y operación unitaria. Pueden identificarse también en regularidades que se observan en el funcionamiento del sistema, tales como: la dinámica de uso y obsolescencia de la colección, así como la variación de su comportamiento, según condicionantes como los recursos financieros, humanos y materiales.

Al tomar a la obsolescencia como una de las regularidades de la información científica y técnica que más afecta a un sistema bibliotecario y de información, se presentan a continuación algunas formulaciones:

1. La obsolescencia depende de la edad de los documentos, del uso de los documentos, del crecimiento de la literatura.

2. Si el uso del documento aumenta, la circulación de éste aumenta (dentro de la biblioteca).

3. Si la obsolescencia es baja, el uso es alto.

4. Si el uso es bajo, la circulación puede ser baja.

La regularidad de la obsolescencia influye en el proceso de circulación del documento.

Para facilitar la lectura, nos permitimos utilizar símbolos que no se encuentran en el alfabeto del lenguaje, pero que corresponden a las letras iniciales de las palabras principales de los enunciados. D e esta manera tenemos: 
$\mathrm{E}_{\mathrm{a}}=$ La edad del documento aumenta.

$\mathrm{O}_{\mathrm{a}}=\mathrm{La}$ obsolescencia del documento aumenta.

$\mathrm{U}_{\mathrm{a}}=\mathrm{El}$ uso del documento aumenta.

$\mathrm{Crec}_{\mathrm{a}}=\mathrm{El}$ crecimiento de la literatura aumenta.

$\mathrm{E}_{\mathrm{b}}=\mathrm{La}$ edad del documento disminuye.

$\mathrm{O}_{\mathrm{b}}=\mathrm{La}$ obsolescencia del documento disminuye

$\mathrm{U}_{\mathrm{b}}=\mathrm{E} l$ uso del documento disminuye

$\mathrm{Crec}_{b}=\mathrm{El}$ crecimiento de la literatura disminuye

$\mathrm{CirC}_{\mathrm{a}}=\mathrm{La}$ circulación del documento aumenta

$\mathrm{Circ}_{b}=\mathrm{La}$ circulación del documento disminuye

\section{A rgumento formalizado}

1. $\left(\left(\mathrm{E}_{\mathrm{a}} \supset \mathrm{O}_{\mathrm{a}}\right) \&\left(\mathrm{U}_{\mathrm{b}} \supset \mathrm{O}_{\mathrm{a}}\right) \&\left(\mathrm{Crec}_{\mathrm{a}} \supset \mathrm{O}_{\mathrm{a}}\right)\right) \&\left(\left(\mathrm{E}_{\mathrm{b}} \supset \mathrm{O}_{\mathrm{b}}\right) \&\left(\mathrm{U}_{\mathrm{a}} \supset \mathrm{O}_{\mathrm{b}}\right) \&\left(\mathrm{Crec}_{\mathrm{b}} \supset\right.\right.$ $\left.\mathrm{O}_{\mathrm{b}}\right)$

2. $\mathrm{U}_{\mathrm{a}} \supset$ Circ.a

3. $\mathrm{O}_{\mathrm{b}} \supset \mathrm{U}_{\mathrm{a}}$

4. $\mathrm{U}_{\mathrm{b}} \supset$ Circ.

\section{$\left(\mathrm{O}_{\mathrm{b}} \supset \mathrm{Circ}_{\mathrm{a}}\right) \&\left(\mathrm{O}_{\mathrm{a}} \supset \mathrm{Circ} . \mathrm{b}\right)$}

\section{Demostración}

\begin{tabular}{|c|c|}
\hline $\begin{array}{l}1\left(\left(E_{a} \supset 0_{a}\right) \&\left(U_{b} \supset 0_{a}\right) \&\right. \\
\left.\left(C e_{a} \supset 0_{a}\right)\right) \&\left(\left(E_{b} \supset 0_{b}\right) \&\right. \\
\left.\left(U_{a} \supset O_{b}\right) \&\left(C r e c b \supset O_{b}\right)\right)\end{array}$ & Premisa \\
\hline 2. $U_{a} \supset$ Cinc.a & Premisa \\
\hline 3. $\mathbf{O}_{\mathrm{b}} \supset \mathrm{U}_{\mathrm{a}}$ & Premisa \\
\hline 4. $\mathrm{U}_{\mathrm{b}} \supset$ Cinc.b & Premisa \\
\hline 5. $0_{a} \equiv \neg 0_{b}$ & $\begin{array}{l}\text { (Señala la relación inversa entre el aumento de la } \\
\text { obsolescencia del documento y su disminución) }\end{array}$ \\
\hline 6. $\mathrm{U}_{\mathrm{a}} \equiv \neg \mathrm{U}_{\mathrm{b}}$ & $\begin{array}{l}\text { (Señala la relación inversa entre el aumento del uso del } \\
\text { documento y su disminución) }\end{array}$ \\
\hline $7.0_{b}$ & Premisa adicional \\
\hline 8. $\left(E_{b} \vee U_{a} \vee C r e c b\right) \supset 0_{b}$ & de 1 por \& e y la equivalencia $((A \supset C) \&(B \supset C)) \equiv((A \vee B) \supset C)$ \\
\hline 9. Ua & de 3 y 7 por $\supset e$ \\
\hline 10 Circ.a & De 2 y 9 por $\supset$ e \\
\hline $11.0_{b} \supset$ Circ.a & de 7-10 por $\supset i$ \\
\hline 12.0a & Premisa adicional \\
\hline 13. $\neg 0 \mathrm{~b}$ & de 5 y 12 por $\equiv$ \\
\hline 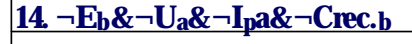 & de 8 y 13 por negación del consecuente y ley de De Morgan \\
\hline 15. $\neg \mathrm{Ua}_{\mathrm{a}}$ & de 14 por \&e \\
\hline 16. Ub & de 6 y 15 por $\equiv$ \\
\hline 17 Circ.b & de 4 y 16 por $\supset$ e \\
\hline $\begin{array}{l}\text { 18. } 0 \mathrm{a} \supset \text { Circ.b } \\
\text { 19 }\left(0_{b} \supset \text { Circ. } a\right) \&\left(0_{a} \supset \text { Circ. } b\right)\end{array}$ & $\begin{array}{l}\text { de } 12-17 \text { por } \supset i \\
\text { de } 11 \text { y } 18 \text { por } \& i\end{array}$ \\
\hline
\end{tabular}


Por último y como un reconocimiento a uno de los bibliotecólogos más destacados en la aplicación de métodos matemáticos y estadísticos en la actividad bibliotecaria, Shiyali Ramamrita Ranganathan (1892 - 1972), se presenta a continuación la formulación lógica de las cinco leyes epónimas (más información al respecto en Ranganathan, 1989) que desde su proposición por este destacado bibliotecólogo hindú han sido reconocidas como los cinco principios generales 0 postulados de la bibliotecología moderna.

\section{LAS CINCO LEYES DE RANGANATHAN}

\section{Los libros son para usarse}

$$
\forall \mathrm{x}(\mathrm{L}(\mathrm{x}) \supset \mathrm{U}(\mathrm{x}))
$$

\section{A cada lector su libro}

$$
\forall \mathrm{x} \forall \mathrm{y}((\mathrm{L}(\mathrm{x}) \& \mathrm{P}(\mathrm{y})) \supset \mathrm{R}(\mathrm{x}, \mathrm{y}))
$$

\section{A cada libro su lector}

$$
\forall \mathrm{x} \exists \mathrm{y}((\mathrm{L}(\mathrm{x}) \& \mathrm{P}(\mathrm{y})) \supset \mathrm{R}(\mathrm{x}, \mathrm{y}))
$$

\section{Ahorre tiempo de los lectores}

(Si es un buen bibliotecario, ahorra el tiempo a los lectores)

$$
\forall \mathrm{x} \forall \mathrm{y}(((\mathrm{B}(\mathrm{x}) \& \mathrm{P}(\mathrm{y})) \supset \mathrm{A}(\mathrm{x}, \mathrm{y})) \supset \mathrm{G}(\mathrm{x}))
$$

\section{La biblioteca es un organismo en crecimiento$$
\forall \mathrm{x}(\mathrm{B}(\mathrm{x}) \supset \mathrm{C}(\mathrm{x}))
$$

\section{CONSIDERACIONES FIN ALES}

Las ciencias bibliotecológica y de la información constituyen un sistema de conocimiento que, al igual como ocurre en otras disciplinas científicas, sirve de soporte teórico a toda una actividad práctica compleja que se rige por principios y condiciones generales, las cuales junto con eventos empíricos concretos representan las premisas que condicionan las relaciones y en ocasiones regularidades de dicha actividad; es decir, las ciencias bibliotecológica y de la información guían a las actividades bibliotecaria y de información y éstas, a su vez, enriquecen a las primeras con su quehacer empírico y cotidiano. 
La lógica, utilizada desde la antigüedad por Aristóteles, en la Grecia clásica para dar explicación a fenómenos complejos y demostrar el carácter científico de determinados saberes, continúa siendo en la actualidad una útil herramienta para tales propósitos.

La formulación lógico-matemática de los principios y relaciones que condicionan como premisas el funcionamiento de las actividades bibliotecarias y de información, no sólo nos permite dar explicación científica a las causas y los efectos que desde el punto de vista teórico la rigen, sino que constituye una valiosa herramienta para pronosticar su comportamiento y como consecuencia enriquecer el lenguaje formal y el cuerpo teórico de las disciplinas bibliológico-informativas.

\section{REFERENCIAS}

Hem pel, Carl G . (1979)- Laexplicacoónaientífica: etudiossobrefilosofíaddlacienaia - Buenos Aires: Paidós.- 485.

Mendelson, Elliott.(1987)- Introdution to mathematical logic- Pascific G rove, Ca.: Wadsworth. - $341 \mathrm{p}$.

Ranganathan, Shiyali Ramamrita. (1989)- Thefivelaus of thelibrary SaienceBangalore:SaradaRanganathanEndowmentforLibraryScience.-449p.

Van-Rijsbergen, C.J. (1996)- "Information, logic, and uncertainty in information Scien ce".- pp.1-10. In:ProceedingCoLIS2.InternationalConferencon ConceptionofLibraryanInformationSaienceIntegrationinPespeetive, 0 c to ber 13 - 16,1996/ PeterIngwersen and NielsO lePors.- Co pen hagen: TheRo yal School of Librarianship.- $484 \mathrm{p}$. 